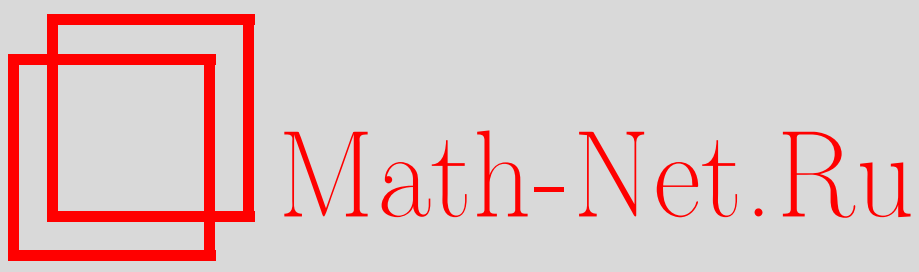

A. Kaźmierczak, Some estimates for the product of modules of specific pairs of foliations, Sibirsk. Mat. Zh., 2020, Volume 61, Number 3, 594-606

DOI: https://doi.org/10.33048/smzh.2020.61.308

Use of the all-Russian mathematical portal Math-Net.Ru implies that you have read and agreed to these terms of use http://www . mathnet.ru/eng/agreement

Download details:

IP: 34.229 .45 .116

April 26, 2023, 03:31:54

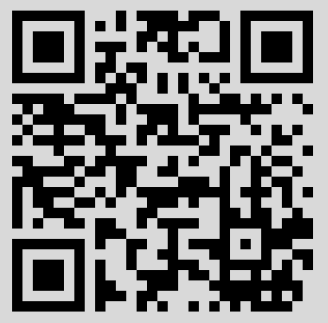


Сибирский математический журнал Май-июнь, 2020. Том 61, № 3

УДК 517.51

\title{
НЕКОТОРЫЕ ОЦЕНКИ ПРОИЗВЕДЕНИЯ МОДУЛЕЙ СПЕЦИАЛЬНОЙ ПАРЫ СЛОЕНИЙ
}

\section{А. Казмирчак}

\begin{abstract}
Аннотация. Рассматривается класс диффеоморфизмов $G=\left(G_{1}, G_{2}\right): M \rightarrow N \subset$ $R_{1} \times R_{2}$, действующих между римановыми многообразиями (где $N$ снабжено метрикой произведения), что позволяет исследовать пары слоений на $M$, состоящих из множеств уровня координат $G_{1}$ и $G_{2}$ соответственно. Приводятся нижние и верхние оценки произведения сопряженных модулей таких пар слоений, зависящие от свойств $N$ и структуры якобиана $J G$. Сформулированы результаты, касающиеся локальных свойств максимальных пар слоений.
\end{abstract}

DOI $10.33048 /$ smzh.2020.61.308

Ключевые слова: слоение, модуль, емкость, субмерсия.

\section{Введение}

Модуль семейства кривых, будучи понятием, взаимным с понятием экстремальной длины, играет существенную роль в теории квазиконформных отображений [1-4]. Он также широко применяется во многих других областях математики таких, как комплексный анализ и анализ на метрических пространствах $[5,6]$ (в частности, на нем основан способ определения пространств Соболева на метрических пространствах с мерой) и геометрическая теория меры [7]. Кроме того, стоит подчеркнуть, что эта характеристика семейства кривых тесно связана с емкостью: $p$-емкость конденсатора равна $p$-модулю всех кривых, входящих в конденсаторы [8-10]. Это понятие обобщено на семейства липшицевых поверхностей в $\mathbb{R}^{n}$ в [11] и далее на $k$-мерные подмногообразия риманова многообразия в [12], тем самым получена возможность изучать модули слоений [13-16].

Пусть $\mathscr{F}-k$-мерное слоение на римановом многообразии $M$ из $C^{1}$ (см. определение 1). Пусть $p>0 . p$-Модулем слоения $\mathscr{F}$ называется число

$$
\bmod _{p}(\mathscr{F})=\inf _{f \in \operatorname{adm}_{p}(\mathscr{F})}\|f\|_{L^{p}(M)},
$$

где $\operatorname{adm}_{p}(\mathscr{F})$ - множество всех $p$-допустимых функций, т. е. п. в. неотрицательных $L^{p}(M)$-интегрируемых функций таких, что $\int_{L} f \geq 1$ для п. в. $L \in \mathscr{F}$. Кроме того, будем считать, что $\bmod _{p}(\mathscr{F})=\infty$, если $\operatorname{adm}_{p}(\mathscr{F})=\varnothing$. Функция $f \in \operatorname{adm}_{p}(\mathscr{F})$ называется $p$-экстремальной, если

$$
\|f\|_{L^{p}(M)}=\bmod _{p}(\mathscr{F}) .
$$

В контексте модулей наиболее эффективно изучение слоений, заданных субмерсиями (см. определение 2), поскольку в этом случае можно использовать явную формулу для $p$-модуля и $p$-экстремальной функции (см. [16, теорема 1]).

(c) 2020 Казмирчак А. 
В данной статье будем рассматривать пары слоений, связанные диффеоморфизмом

$$
G=\left(G_{1}, G_{2}\right): M \rightarrow R_{1} \times R_{2},
$$

действующим на произведении римановых многообразий (с метрикой произведения). Согласно определению 3 такие слоения состоят из множеств уровня соответствующих координат функций из $G$. Такую конструкцию можно считать обобщением четырехсторонника с двумя слоениями, соединяющими противоположные пары его сторон. Плоский четырехсторонник определяется в [1] как открытое множество $Q \subset \mathbb{R}^{2}$, гомеоморфное прямоугольнику, с двумя различными непересекающимися дугами на его границе. Если две такие дуги определены, то можно рассматривать на $Q$ семейства кривых, соединяющих эти дуги. Кроме того, естественным будет изучение модуля множества всех кривых с таким свойством. Альфорс в [1] показал, что для каждого четырехсторонника произведение 2-модулей таких семейств равно единице. В свою очередь, А. С. Романов в [17] доказал аналогичный результат для произведение модулей с сопряженными степенями суммируемости. Последнее утверждение вместе с монотонностью модуля (см. свойство 1 теоремы 7) делает интуитивно понятной идею теоремы 3 , в которой утверждается, что для пары слоений $\mathscr{F}_{1}, \mathscr{F}_{2}$, связанных диффеоморфизмом $\left(G ; R_{1}, R_{2}\right)$, произведение

$$
\bmod _{p_{1}}\left(\mathscr{F}_{1}\right) \cdot \bmod _{p_{2}}\left(\mathscr{F}_{2}\right), \quad \text { где } \frac{1}{p_{1}}+\frac{1}{p_{2}}=1,
$$

не превосходит 1. Следствие 2, будучи эквивалентом теоремы 4.3 из [14], дает достаточное условие максимальности значения произведения сопряженных модулей (которое равно единице) для слоений вышеуказанного типа. Помимо требований теоремы 3 в следствии 2 дополнительно предполагается особое строение якобиана $J G$, а именно

$$
\begin{gathered}
J G=J G_{\mathscr{F}_{1}} \cdot J G_{\mathscr{F}_{2}} \quad\left(\text { эквивалентно } \mathscr{F}_{1} \perp \mathscr{F}_{2}(\text { см. лемму } 1)\right), \\
c_{1} J G_{\mathscr{F}_{1}}^{p_{1}}=c_{2} J G_{\mathscr{F}_{2}}^{p_{2}} \text { для некоторых } c_{1}, c_{2}>0 .
\end{gathered}
$$

Легко убедиться в том, что гёльдерова зависимость между экстремальными функциями (с подходящей константой, как в (15)) является достаточным условием равенства единице произведения (1) для любой пары слоений из рассматриваемого класса. Примечательно, что это требование оказывается и необходимым условием равенства данного произведения единице (см. теорему 4).

С другой стороны, рассматривая более широкий класс диффеоморфизмов, чем в теореме 3 , т. е. все $G: M \rightarrow N \subset R_{1} \times R_{2}$, действующие на открытых подмножествах $N$ из $R_{1} \times R_{2}$, и в то же время накладывая условия (2), приходим к заключению, что вопреки утверждению теоремы 3 произведение (1) должно не превосходить единицу (см. теорему 2 ).

В заключение в разд. 3 изучим некоторые локальные свойства максимальной пары слоений. Оказывается, такие слоения обладают свойством «локальной максимальности» в том смысле, что для любого открытого множества $M^{\prime}$, вырезанного из $M$ вдоль его листов,

$$
\bmod _{p_{1}}\left(\left.\mathscr{F}_{1}\right|_{M^{\prime}}\right) \cdot \bmod _{p_{2}}\left(\left.\mathscr{F}_{2}\right|_{M^{\prime}}\right)=1
$$

при условии, что произведение (1) равно 1 (следствие 4). Кроме того, $p$-экстремальная функция максимального слоения «локально экстремальна». А именно, если через $f$ обозначим $p$-экстремальную функцию слоения $\mathscr{F}$, определенного на $M$, а через $f^{\prime}-p$-экстремальную функцию $\left.\mathscr{F}\right|_{M^{\prime}}$, то $f^{\prime}=c \cdot f$, где 
$c>0$ - постоянная, зависящая от норм $f$ и $f^{\prime}$ (см. теорему 8). Эти локальные свойства вполне согласуются с результатами, касающимися емкостей, если $M-$ открытое подмножество $\mathbb{R}^{2}$ (ср. [17]).

\section{1. Модуль слоения, заданного субмерсией}

ОПРЕДЕЛЕНИЕ $1 . k$-Мерным слоением $\mathscr{F}$ на $M$ называется такое семейство попарно не пересекающихся $k$-мерных подмногообразий $M$, что $M=\bigcup_{L \in \mathscr{F}} L$ и для любой точки $x \in M$ существуют окрестность $D$ и отображение $\varphi=$ $\left(\varphi^{1}, \varphi^{2}, \ldots, \varphi^{n}\right): D \rightarrow \mathbb{R}^{n}$ такие, что $\varphi_{i}(D)=(0,1), i=1, \ldots, n$, и для любой компоненты связности $P$ любого $L \cap D$

$$
\varphi_{\mid P}^{k+1}=\text { const }, \ldots, \varphi_{\mid P}^{n}=\text { const. }
$$

Через $L^{x}$ обозначим лист $\mathscr{F}$, проходящий через $x \in M$.

Заметим, что в определении 1 листы не обязательно связны.

Пусть $M$ и $P$ - римановы многообразия. Напомним, что гладкое отображение $\phi: M \rightarrow P$ называется субмерсией, если для всех $x \in M$ его дифференциал $\phi_{*}(x): T_{x} M \rightarrow T_{\phi(x)} P$ сюръективен. Якобианом $J_{\phi}$ является функция, сопоставляющая каждой точке $x \in M$ якобиан изоморфизма $\left.\phi_{*}(x)\right|_{\operatorname{ker}\left(\phi_{*}(x)\right)^{\perp}}$.

ОПРЕДЕЛЕНИЕ 2. Будем говорить, что слоение $\mathscr{F}=\left\{\phi^{-1}(y): y \in \phi(M)\right\}$ задано субмерсией $\phi$.

В теореме 1 (см. [16]) приводятся формулы для $p$-модуля и $p$-экстремальной функции слоения, заданного субмерсией. Она будет часто применяться в данной статье.

Теорема 1 [16]. Если слоение $\mathscr{F}$ задано субмерсией $\phi: M \rightarrow P$ и $\int_{L} J_{\phi}^{\frac{1}{p-1}}<$ $\infty$ для п. в. $L \in \mathscr{F}$, то при $p>1$

$$
\bmod _{p}(\mathscr{F})=\left(\int_{\phi(M)}\left(\int_{L^{x}} J_{\phi}^{\frac{1}{p-1}}\right)^{1-p}\right)^{\frac{1}{p}} .
$$

Кроме того, если $\bmod _{p}(\mathscr{F})<\infty$, то существует единственная $p$-экстремальная функция, которая задается формулой

$$
f(x)=\frac{J_{\phi}(x)^{\frac{1}{p-1}}}{\int_{L^{x}} J_{\phi}^{\frac{1}{p-1}}} .
$$

Непосредственно из равенства (4) вытекает

Следствие 1. Если $f-p$-экстремальная функция слоения $\mathscr{F}$, заданного субмерсией, то $\int_{L^{x}} f=1$ для всех $x \in M$.

\section{2. Оценки для произведения модулей слоений}

Отметим, что для любых диффеоморфизма $G: M \rightarrow N$ между римановыми многообразиями и слоения $\mathscr{F}$ на $M$

$$
G(\mathscr{F})=\{G(L): L \in \mathscr{F}\}
$$


является слоением на $N$. Через $J G_{\mathscr{F}}(x)$ будем обозначать якобиан изоморфизма $\left.G_{*}(x)\right|_{T_{x} L^{x}}$, а через $J G_{\mathscr{F} \perp}(x)$ - якобиан изоморфизма $\left.G_{*}(x)\right|_{\left(T_{x} L^{x}\right)^{\perp}}$.

Всюду в статье предполагаем, что $R_{1} \times R_{2}$ снабжено метрикой произведения, а $N \subset R_{1} \times R_{2}-$ метрикой, индуцированной из $R_{1} \times R_{2}$.

ОПРЕДЕЛЕНИЕ 3 . Пусть $G: M \rightarrow N \subset R_{1} \times R_{2}$ - диффеоморфизм. Определим $\mathscr{F}_{1}$ как слоение, заданное $G_{2}$, а $\mathscr{F}_{2}-$ как слоение, заданное $G_{1}$. Назовем эту пару системой слоений, свлзанных диффеоморфизмом $\left(G ; R_{1}, R_{2}\right)$. $J G_{1}$.

Таким образом, для рассматриваемых слоений $J G_{\mathscr{F}_{1}^{\perp}}=J G_{2}$ и $J G_{\mathscr{F}_{2}^{\perp}}=$

Лемма 1. Пусть $G: M \rightarrow N \subset R_{1} \times R_{2}$ - диффеоморфизм и $\mathscr{F}_{1}, \mathscr{F}_{2}$ связаны диффеоморфизмом $\left(G ; R_{1}, R_{2}\right)$. Тогда

$$
J G_{\mathscr{F}_{1}} \cdot J G_{\mathscr{F}_{2}} \leq J G
$$

где равенство достигается в том и в только том случае, когда слоения ортогональны.

ОПРЕДЕЛЕниЕ 4 . Вещественные числа $p_{1}, p_{2}>1$ будем называть сопряженными, если $\frac{1}{p_{1}}+\frac{1}{p_{2}}=1$.

Теорема 2. Пусть $R_{1}, R_{2}$ имеют конечный объем, $G: M \rightarrow N \subset R_{1} \times R_{2}-$ диффеоморфизм и $\mathscr{F}_{1}, \mathscr{F}_{2}-$ слоения, связанные диффеоморфизмом $\left(G ; R_{1}, R_{2}\right)$. Если $\mathscr{F}_{1} \perp \mathscr{F}_{2}$ и для пары сопряженных $p_{1}, p_{2}$ и некоторых $0<c_{1}, c_{2} \in \mathbb{R}$

$$
c_{1} J G_{\mathscr{F}_{1}}^{p_{1}}(x)=c_{2} J G_{\mathscr{F}_{2}}^{p_{2}}(x) \text { для всех } x \in M,
$$

то $\bmod _{p_{1}}\left(\mathscr{F}_{1}\right) \cdot \bmod _{p_{2}}\left(\mathscr{F}_{2}\right) \geq 1$.

ДокАЗАтЕЛЬСтво. Обозначим через $\mu_{R_{i}}, i=1,2$, меру Лебега, определенную на $R_{i}$. Поскольку на $R_{1} \times R_{2}$ введена метрика произведения, $J \pi_{i}=1$ $\left(\pi_{i}: R_{1} \times R_{2} \rightarrow R_{i}\right)$. Таким образом,

$$
\int_{L_{i}^{x}} J G_{\mathscr{F}_{i}}=\int_{G\left(L_{i}^{x}\right)} J \pi_{i}=\int_{G_{i}\left(L_{i}^{x}\right)} 1 \leq \mu_{R_{i}}\left(R_{i}\right) .
$$

Кроме того, из сопряженности $p_{1}$ и $p_{2}$ следует, что

$$
p_{2}-1=\frac{p_{1}}{p_{2}}=\frac{1}{p_{1}-1}
$$

откуда в силу (5) имеем

$$
J G_{\mathscr{F}_{2}}^{p_{2}-1}=J G_{\mathscr{F}_{2}}^{\frac{p_{2}}{p_{1}}}=\left(\frac{c_{1}}{c_{2}}\right)^{\frac{1}{p_{1}}} J G_{\mathscr{F}_{1}} .
$$

Кроме того, из ортогональности слоений получаем

$$
J G_{\mathscr{F}_{1}^{\perp}}=J G_{\mathscr{F}_{2}}, \quad J G_{\mathscr{F}_{2}^{\perp}}=J G_{\mathscr{F}_{1}} .
$$

Таким образом, для всех $x \in M$

$$
\begin{aligned}
\int_{L_{1}^{x}} J G_{\mathscr{F}_{1}^{\perp}}^{\frac{1}{p_{1}-1}}=\int_{L_{1}^{x}} J G_{\mathscr{F}_{2}}^{\frac{1}{p_{1}-1}}= & \int_{G_{1}\left(L_{1}^{x}\right)}\left(J G_{\mathscr{F}_{2}}^{\frac{1}{p_{1}-1}} \cdot J G_{\mathscr{F}_{1}}^{-1}\right) \circ G^{-1} \\
& =\int_{G_{1}\left(L_{1}^{x}\right)}\left(\frac{c_{1}}{c_{2}}\right)^{\frac{1}{p_{1}}} 1=\left(\frac{c_{1}}{c_{2}}\right)^{\frac{1}{p_{1}}} \mu_{R_{1}}\left(G_{1}\left(L_{1}^{x}\right)\right)<\infty .
\end{aligned}
$$


Аналогично $\int_{L_{2}^{x}} J G_{\mathscr{F}_{2}^{\perp}}^{\frac{1}{p_{2}-1}}<\infty$, а значит, все предположения теоремы 1 выполнены. Тем самым, применяя (3), (9), (7), (8) и (6), получаем

$$
\begin{aligned}
\bmod _{p_{1}}^{p_{1}}\left(\mathscr{F}_{1}\right)= & \int_{R_{2}}\left(\int_{L_{1}} J G_{\mathscr{F}_{2}}^{p_{2}-1}\right)^{1-p_{1}}=\left(\frac{c_{1}}{c_{2}}\right)^{-\frac{1}{p_{2}}} \int_{R_{2}}\left(\int_{L_{1}} J G_{\mathscr{F}_{1}}\right)^{1-p_{1}} \\
& \geq\left(\frac{c_{1}}{c_{2}}\right)^{-\frac{1}{p_{2}}} \int_{R_{2}} \mu_{R_{1}}\left(R_{1}\right)^{1-p_{1}}=\left(\frac{c_{1}}{c_{2}}\right)^{-\frac{1}{p_{2}}} \mu_{R_{2}}\left(R_{2}\right) \mu_{R_{1}}\left(R_{1}\right)^{1-p_{1}} .
\end{aligned}
$$

Аналогично

$$
\bmod _{p_{2}}^{p_{2}}\left(\mathscr{F}_{2}\right) \geq\left(\frac{c_{2}}{c_{1}}\right)^{-\frac{1}{p_{1}}} \mu_{R_{1}}\left(R_{1}\right) \mu_{R_{2}}\left(R_{2}\right)^{1-p_{2}}
$$

Таким образом,

$$
\begin{aligned}
\bmod _{p_{1}}\left(\mathscr{F}_{1}\right) \bmod _{p_{2}}\left(\mathscr{F}_{2}\right) \geq\left(\frac{c_{1}}{c_{2}}\right)^{-\frac{1}{p_{1} \cdot p_{2}}} \mu_{R_{2}}\left(R_{2}\right)^{\frac{1}{p_{1}}} \mu_{R_{1}}\left(R_{1}\right)^{\frac{1}{p_{1}}-1} \\
\cdot\left(\frac{c_{1}}{c_{2}}\right)^{\frac{1}{p_{1} \cdot p_{2}}} \mu_{R_{1}}\left(R_{1}\right)^{\frac{1}{p_{2}}} \mu_{R_{2}}\left(R_{2}\right)^{\frac{1}{p_{2}}-1}=1 .
\end{aligned}
$$

Следствие 2. Если $N=R_{1} \times R_{2}$ в условии теоремы 2, то ее утверждение усиливается:

$$
\bmod _{p_{1}}\left(\mathscr{F}_{1}\right) \cdot \bmod _{p_{2}}\left(\mathscr{F}_{2}\right)=1 .
$$

ДокАЗАтЕЛьство. В случае, когда $N=R_{1} \times R_{2}$, имеем $G_{i}\left(L_{i}^{x}\right)=R_{i}$, так что $\int_{L_{i}^{x}} J G_{\mathscr{F}_{i}}=\mu_{R_{i}}\left(R_{i}\right)$. Тем самым все существенные неравенства после $(6)$ могут быть заменены равенствами, что приводит к требуемому утверждению.

ПримеР 1. Пусть $R_{1}, R_{2} \subset \mathbb{R}$ - открытые промежутки, $G=\left.\mathrm{id}\right|_{N}$, где $N \subset R_{1} \times R_{2}$ - ограниченная область. Обозначим через $\mathscr{F}_{1}, \mathscr{F}_{2}$ слоения, связанные диффеоморфизмом $\left(G ; R_{1}, R_{2}\right)$. Эти ортогональные слоения состоят из горизонтальных и вертикальных отрезков соответственно (или, возможно, из сумм таких отрезков). Поскольку $J G_{\mathscr{F}_{1}}=J \pi_{2}=1$ и $J G_{\mathscr{F}_{2}}=J \pi_{1}=1$, предположения теоремы 2 выполнены для любой пары $p_{1}, p_{2}$ сопряженных чисел. Стало быть,

$$
\bmod _{p_{1}} \mathscr{F}_{1} \cdot \bmod _{p_{2}} \mathscr{F}_{2} \geq 1
$$

ПримеР 2 (сектор классического кольца). Пусть $a, b>0$ таковы, что $a<b$ и $\alpha \in(0,2 \pi)$. Положим

$$
M=\left\{(x, y) \in \mathbb{R}^{2}: a<r(x, y)<b, 0<\theta(x, y)<\alpha\right\},
$$

где $r(x, y)$ и $\theta(x, y)$ - полярные координаты точки $(x, y)$. Введем на $M$ евклидову метрику, индуцированную из $\mathbb{R}^{2}$. Пусть $G: M \rightarrow(\ln (a), \ln (b)) \times(0, \alpha)$ определено по формуле

$$
G(x, y)=(\ln (r(x, y)), \theta(x, y)) .
$$

Обозначим через $\mathscr{F}_{1}, \mathscr{F}_{2}$ систему слоений, связанных диффеоморфизмом $(G ;(\ln (a), \ln (b)),(0, \alpha))$. Другими словами,

$$
\begin{gathered}
\mathscr{F}_{1}=\left\{G^{-1}((a, b) \times\{\theta\}): \theta \in(0, \alpha)\right\}, \\
\mathscr{F}_{2}=\left\{G^{-1}(\{z\} \times(0, \alpha)): z \in(\ln (a), \ln (b))\right\} .
\end{gathered}
$$


Тогда

$$
J G_{\mathscr{F}_{1}}=J G_{\mathscr{F}_{2}}=\frac{1}{r}
$$

в то время как $J G=\frac{1}{r^{2}}$. Отсюда $\mathscr{F}_{1} \perp \mathscr{F}_{2}$ (см. лемму 1$)$. Тем самым по следствию 2 равенство (1) будет выполнено, если $p_{1}=p_{2}=2$. Действительно, по формуле (3) получаем $\bmod _{2}^{2}\left(\mathscr{F}_{1}\right)=\alpha \ln \left(\frac{b}{a}\right)^{-1}$ и $\bmod _{2}^{2}\left(\mathscr{F}_{2}\right)=\alpha^{-1} \ln \left(\frac{b}{a}\right)$. Значит, (1) выполнено.

Лемма 2. Пусть $p_{1}, p_{2}-$ сопряженные числа и $\mathscr{F}_{1}, \mathscr{F}_{2}-$ пара слоений, связанных диффеоморфизмом $G: M \rightarrow R_{1} \times R_{2}$, такие, что $\int_{L_{1}} J G_{\mathscr{F}_{1}^{\perp}}^{\frac{1}{p_{1}-1}}<\infty$ для всех $L_{1} \in \mathscr{F}_{1}$. Если $f_{1}-p_{1}$-экстремальная функция слоения $\mathscr{F}_{1}$, то для всех $L_{2} \in \mathscr{F}_{2}$

$$
\int_{L_{2}} \bar{f}_{2} \geq 1, \quad \text { где } \bar{f}_{2}=\left\|f_{1}\right\|_{L^{p_{1}(M)}}^{-p_{1}} f_{1}^{p_{1}-1} .
$$

ДокАЗАТЕЛЬство. Имеем $J G=J G_{\mathscr{F} \perp} \cdot J G_{\mathscr{F}}$ и $J G \geq J G_{\mathscr{F}_{1}} \cdot J G_{\mathscr{F}_{2}}$ (ср. с леммой 1). Значит, $J G_{\mathscr{F} \perp} \geq J G_{\mathscr{F}}$. Применяя формулу (4), получим

$$
f_{1}=\frac{J G_{\mathscr{F} \perp}^{\frac{1}{p_{1}-1}}}{\int_{L_{1}} J G_{\mathscr{F} 1}^{\frac{1}{p_{1}-1}}} \geq \frac{J G_{\mathscr{F}_{2}}^{\frac{1}{p_{1}-1}}}{\int_{L_{1}} J G_{\mathscr{F} \perp}^{\frac{1}{p_{1}-1}}} .
$$

Стало быть, $f_{1}^{p_{1}-1} \geq \frac{J G_{\mathscr{F}_{2}}}{\left(\int_{L_{1}} J G_{\mathscr{F}_{1}^{\perp}}^{\frac{1}{p_{1}-1}}\right)^{p_{1}-1}}$, откуда ввиду $(3)$ для любого $L_{2} \in \mathscr{F}_{2}$ имеем

$$
\begin{aligned}
\int_{L_{2}} \bar{f}_{2}=\left\|f_{1}\right\|_{L^{p_{1}}(M)}^{-p_{1}} \int_{L_{2}} f_{1}^{p_{1}-1} \geq\left\|f_{1}\right\|_{L^{p_{1}}(M)}^{-p_{1}} \int_{R_{2}}\left(\int_{L_{1}} J G_{\mathscr{F}_{1}^{\perp}}^{\frac{1}{p_{1}-1}}\right)^{1-p_{1}} \\
=\left\|f_{1}\right\|_{L^{p_{1}(M)}}^{-p_{1}}\left\|f_{1}\right\|_{L^{p_{1}(M)}}^{p_{1}}=1 .
\end{aligned}
$$

Лемма 3. Если выполнены предположения леммы 2 (и $\bar{f}_{2}$ определена, как в лемме 2), то $\left\|f_{1}\right\|_{L^{p_{1}(M)}}\left\|\bar{f}_{2}\right\|_{L^{p_{2}(M)}}=1$.

ДокАЗАТЕЛЬСтво. Из (7) получаем

$$
\begin{aligned}
& \left\|f_{1}\right\|_{L^{p_{1}(M)}}\left\|\bar{f}_{2}\right\|_{L^{p_{2}(M)}}=\left\|f_{1}\right\|_{L^{p_{1}(M)}} \frac{\left\|f_{1}^{p_{1}-1}\right\|_{L^{p_{2}(M)}}}{\left\|f_{1}\right\|_{L^{p_{1}}(M)}^{p_{1}}} \\
& =\left\|f_{1}\right\|_{L^{p_{1}(M)}}^{1-p_{1}}\left(\int_{M} f_{1}^{\left(p_{1}-1\right) \frac{p_{1}}{p_{1}-1}}\right)^{\frac{1}{p_{2}}}=\left\|f_{1}\right\|_{L^{p_{1}}(M)}^{1-p_{1}}\left\|f_{1}\right\|_{L^{p_{1}(M)}}^{\frac{p_{1}}{p_{2}}}=1 .
\end{aligned}
$$

Теорема 3. Пусть $\mathscr{F}_{1}, \mathscr{F}_{2}$ - пара слоений, связанных диффеоморфизмом $G: M \rightarrow R_{1} \times R_{2}$, и $p_{1}, p_{2}-$ сопряженные числа. Пусть $\int_{L_{1}} J G_{\mathscr{F}_{1}^{\perp}}^{\frac{1}{p_{1}-1}}<\infty$ для всех $L_{1} \in \mathscr{F}_{1}$ и $\mathscr{F}_{1}$ имеет $p_{1}$-экстремальную функцию. Тогда

$$
\bmod _{p_{1}}\left(\mathscr{F}_{1}\right) \cdot \bmod _{p_{2}}\left(\mathscr{F}_{2}\right) \leq 1
$$


ДокАЗАТЕЛЬСтво. Обозначим через $f_{1} p_{1}$-экстремальную функцию $\mathscr{F}_{1}$. В силу лемм 2 и $3 \bar{f}_{2}=\left\|f_{1}\right\|_{p_{1}}^{-p_{1}} f_{1}^{p_{1}-1} p_{2}$-допустима, так что

$$
\bmod _{p_{2}}\left(\mathscr{F}_{2}\right) \leq\left\|\bar{f}_{2}\right\|_{L^{p_{2}(M)}} .
$$

По лемме $3\left\|f_{1}\right\|_{L^{p_{1}(M)}}\left\|\bar{f}_{2}\right\|_{L^{p_{2}(M)}}=1$, что вместе с (13) приводит к требуемомy.

ОПРЕДЕЛЕНИЕ 5. Пусть $G: M \rightarrow R_{1} \times R_{2}$ - диффеоморфизм. Назовем пару $\mathscr{F}_{1}, \mathscr{F}_{2}$, связанную диффеоморфизмом $\left(G ; R_{1}, R_{2}\right),\left(p_{1}, p_{2}\right)$-максималъной, если числа $p_{1}, p_{2}$ сопряженные и

$$
\bmod _{p_{1}}\left(\mathscr{F}_{1}\right) \cdot \bmod _{p_{2}}\left(\mathscr{F}_{2}\right)=1 .
$$

Теорема 4. Пусть числа $p_{1}, p_{2}$ сопряженные, а $\mathscr{F}_{1}, \mathscr{F}_{2}-$ пара $\left(p_{1}, p_{2}\right)$ максимальных слоений, связанных диффеоморфизмом $\left(G ; R_{1}, R_{2}\right)$. Кроме того, пусть

$$
\int_{L_{1}} J G_{\mathscr{F}_{1}^{\perp}}^{\frac{1}{p_{1}-1}}<\infty \quad \text { для всех } L_{1} \in \mathscr{F}_{1} .
$$

Тогда

$$
f_{2}=\frac{f_{1}^{p_{1}-1}}{\left\|f_{1}\right\|_{L^{p_{1}}(M)}^{p_{1}}}
$$

где $f_{1}$ и $f_{2}-p_{1}$ - и $p_{2}$-экстремальные функции для $\mathscr{F}_{1}$ и $\mathscr{F}_{2}$ соответственно.

ДоКАЗАТЕЛЬСтво. В силу максимальности данной пары слоений (см. определение 5) очевидно, что

$$
\bmod _{p_{i}}\left(\mathscr{F}_{i}\right)<\infty \quad \text { для } i=1,2 .
$$

Поскольку (14) выполнено, можно воспользоваться теоремой 1 и, опираясь на (16), получить существование $f_{1}$. Согласно леммам 2 и 3 функция

$$
\bar{f}_{2}=\left\|f_{1}\right\|_{L^{p_{1}(M)}}^{-p_{1}} f_{1}^{p_{1}-1}
$$

$p_{2}$-допустима для $\mathscr{F}_{2}$ и $\left\|\bar{f}_{2}\right\|_{L^{p_{2}(M)}}=\left\|f_{1}\right\|_{L^{p_{1}}(M)}^{-1}$. Отсюда (снова в силу предположения о максимальности) $\left\|\bar{f}_{2}\right\|_{L^{p_{2}(M)}}=\left\|f_{2}\right\|_{L^{p_{2}(M)}}$. Из единственности экстремальной функции получаем $f_{2}=\bar{f}_{2}$, что и требовалось доказать.

Этот результат может быть также получен с помощью другого, вариационного, подхода как следствие теоремы 3 и теоремы 4.5 из [13].

Теорема 4 позволяет установить следующее важное свойство максимального слоения.

Теорема 5. Пусть $\mathscr{F}_{1}, \mathscr{F}_{2}$ - слоения, связанные диффеоморфизмом $\left(G ; R_{1}, R_{2}\right)$, а числа $p_{1}, p_{2}$ сопряженные. Если пара $\mathscr{F}_{1}, \mathscr{F}_{2}\left(p_{1}, p_{2}\right)$-максимальна и

$$
\int_{L_{1}} J G_{\mathscr{F}_{1}^{\perp}}^{\frac{1}{p_{1}-1}}<\infty \quad \text { для всех } L_{1} \in \mathscr{F}_{1},
$$

Tо $\mathscr{F}_{1} \perp \mathscr{F}_{2}$.

ДокАЗАТЕЛЬСтво. Напомним, что по теореме 4 экстремальные функции $f_{1}$ и $f_{2}$ для максимальных слоений существуют и $f_{2}=\frac{f_{1}^{p_{1}-1}}{\left\|f_{1}\right\|_{\left.L^{p_{1}} p_{1}\right)}^{p_{1}}}$. 
Предположим, что слоения не ортогональны в некоторой точке $x \in M$. Тогда $J G(x)>J G_{\mathscr{F}_{1}}(x) \cdot J G_{\mathscr{F}_{2}}(x)$ (т. е. $\left.J G_{\mathscr{F}_{1}^{\perp}}(x)>J G_{\mathscr{F}_{2}}(x)\right)$. Гладкость $G$ влечет существование открытой окрестности $L_{2}^{\prime} \subset L_{2}^{x}$ точки $x$ такой, что последнее неравенство выполнено для всех $y \in L_{2}^{\prime}$. Тогда $\int_{L_{2}^{\prime}} J G_{\mathscr{F}_{1}^{\perp}}>\int_{L_{2}^{\prime}} J G_{\mathscr{F}_{2}}$, а также $\int_{L_{2}^{x}} J G_{\mathscr{F}_{1}^{\perp}}>\int_{L_{2}^{x}} J G_{\mathscr{F}_{2}}$ (поскольку $J G_{\mathscr{F}_{1}^{\perp}} \geq J G_{\mathscr{F}_{2}}$ всюду). Следовательно, $\int_{L_{2}^{x}} f_{2}>1$. Однако, с другой стороны, $\int_{L_{2}} f_{2}=1$ для всех $L_{2} \in \mathscr{F}_{2}$ (см. следствие 1); противоречие.

В следующей теореме сформулируем необходимое и достаточное условия максимальности пары слоений.

Теорема 6. Пусть числа $p_{1}, p_{2}$ сопряженные и $\mathscr{F}_{1}, \mathscr{F}_{2}$ связаны диффеоморфизмом $\left(G ; R_{1}, R_{2}\right)$. Допустим, что

$$
\int_{L_{1}} J G_{\mathscr{F}_{1}^{\perp}}^{\frac{1}{p_{1}-1}}<\infty \quad \text { для всех } L_{1} \in \mathscr{F}_{1} .
$$

Тогда пара $\mathscr{F}_{1}, \mathscr{F}_{2}\left(p_{1}, p_{2}\right)$-максимальна в том и только том случае, когда $\mathscr{F}_{1} \perp \mathscr{F}_{2}$ и существует положительная постоянная $c$ такая, что

$$
f_{2}=c f_{1}^{p_{1}-1}
$$

где $f_{1}$ и $f_{2}-p_{1}$ - и $p_{2}$-экстремальные функции для $\mathscr{F}_{1}$ и $\mathscr{F}_{2}$ соответственно.

ДокАЗАТЕЛЬСтво. « $\Rightarrow$ Следует напрямую из теорем 4 и 5.

«ÆВ В силу $(20)$, теоремы 1 и ортогональности слоений выполнена следующая цепочка равенств:

$$
\begin{aligned}
1=\int_{L_{2}} f_{2}=c \int_{L_{2}} f_{1}^{p_{1}-1}=c \int_{L_{2}} \frac{J G_{\mathscr{F}_{2}}}{\left(\int_{L_{1}} J G_{\mathscr{F}_{2}}^{\frac{1}{p_{1}-1}}\right)^{p_{1}-1}} \\
\quad=c \int_{R_{2}}\left(\int_{L_{1}} J G_{\mathscr{F}_{2}}^{\frac{1}{p_{1}-1}}\right)^{1-p_{1}}=c\left\|f_{1}\right\|_{L^{p_{1}}(M)}^{p_{1}},
\end{aligned}
$$

стало быть, $c=\left\|f_{1}\right\|_{L^{p_{1}}(M)}^{-p_{1}}$. Таким образом, $f_{2}=\frac{f_{1}^{p_{1}-1}}{\left\|f_{1}\right\|_{L^{p_{1}}(M)}^{p_{1}}}$. Утверждение теоремы следует из леммы 3.

ПримеР 3. Найдем экстремальные функции для слоений из примера 2. Для этого, возможно, будет удобнее использовать полярные координаты, т. е. $\widetilde{G}(x, y)=(r(x, y), \theta(x, y))$. Очевидно, что $J \widetilde{G}_{\mathscr{F}_{1}}=1$ и $J \widetilde{G}_{\mathscr{F}_{2}}=\frac{1}{r}$. Обозначим через $f_{1} p_{1}$-экстремальную функцию для $\mathscr{F}_{1}$, а через $f_{2}-p_{2}$-экстремальную функцию для $\mathscr{F}_{2}$. Поскольку $\mathscr{F}_{1}, \mathscr{F}_{2}$ связаны (также) диффеоморфизмом $(\widetilde{G} ;(a, b),(0, \alpha))$, то, применяя формулу $(4)$, получим

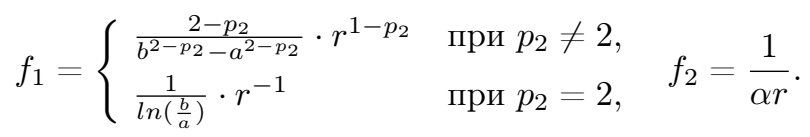

Тем самым $\frac{1}{\alpha r}=f_{2}=c f_{1}^{p_{1}-1}$, что совпадает с соотношением (20), которое согласно теореме 6 означает $\left(p_{1}, p_{2}\right)$-максимальность слоений $\mathscr{F}_{1}, \mathscr{F}_{2}$ (для любой 
пары $\left(p_{1}, p_{2}\right)$ сопряженных чисел, а не только для $p_{1}=p_{2}=2$, что уже было показано в примере 2).

Заметим, что импликация, обратная к импликации теоремы 5 , неверна, поскольку произведение сопряженных модулей двух ортогональных слоений строго меньше единицы.

ПримеР 4. Рассмотрим диффеоморфизм $G:(x, y) \in \mathbb{R} \mapsto\left(x^{2}-y^{2}, x y\right)$ и многообразие $M=G^{-1}\left((0,1)^{2}\right)$. Пусть $\mathscr{F}_{1}, \mathscr{F}_{2}$ - пара слоений, связанных диффеоморфизмом $(G ;(0,1),(0,1))$ (ср. с рассуждениями в [17] относительно $\left(p_{1}, p_{2}\right)$-емкостей двух конденсаторов на четырехстороннике, определенном на $\left.M=G^{-1}\left((0,1)^{2}\right)\right)$. Ясно, что $J G=2 r^{2}$, в то время как $J G_{\mathscr{F}_{1}}=2 r$ и $J G_{\mathscr{F}_{2}}=$ $r$. Тогда $J G_{\mathscr{F}_{1}}=2 J G_{\mathscr{F}_{2}}$ и $J G=J G_{\mathscr{F}_{1}} \cdot J G_{\mathscr{F}_{2}}$. Тем самым предположения следствия 2 выполнены для $p_{1}=p_{2}=2$. Следовательно, пара данных слоений $(2,2)$-максимальна.

Однако $\mathscr{F}_{1}, \mathscr{F}_{2}$ не $\left(p_{1}, p_{2}\right)$-максимальны для пары сопряженных $p_{1} \neq p_{2}$. Действительно, обозначим через $\tilde{f}_{1}$ и $\tilde{f}_{2} p_{1}$ - и $p_{2}$-экстремальные функции для $\mathscr{F}_{1}$ и $\mathscr{F}_{2}$ соответственно. Ввиду формулы (4) получаем

$$
\tilde{f}_{1}=\frac{J G_{\mathscr{F}_{2}}^{\frac{1}{p_{1}-1}}}{\int_{L_{1}} J G_{\mathscr{F}_{2}}^{\frac{1}{p_{1}-1}}}=\frac{r^{\frac{1}{p_{1}-1}}}{\int_{L_{1}} r^{\frac{1}{p_{1}-1}}}=\frac{r^{\frac{1}{p_{1}-1}}}{\frac{1}{2} \int_{0}^{1} r^{p_{2}-2} \circ G^{-1} d a} .
$$

Аналогично

$$
\tilde{f}_{2}=\frac{r^{p_{1}-1}}{\int_{0}^{1} r^{p_{1}-2} \circ G^{-1} d b}
$$

где $a=G_{1}(x, y), b=G_{2}(x, y)$. Далее, согласно теореме $6\left(p_{1}, p_{2}\right)$-максимальность влечет, что $\tilde{f}_{2}=c \tilde{f}_{1}^{p_{1}-1}$. Подставляя выражения, полученные выше, в это равенство, имеем

$$
\frac{r^{p_{1}-2}(x, y)}{c \cdot 2^{p_{1}-1}}=\frac{\int_{0}^{1} r^{p_{1}-2} \circ G^{-1} d b}{\left(\int_{0}^{1} r^{p_{2}-2} \circ G^{-1} d a\right)^{p_{1}-1}}
$$

для всех $(x, y) \in M$, что эквивалентно тому, что

$$
\left(a^{2}+4 b^{2}\right)^{\frac{p_{1}-2}{4}}=r(x, y)^{p_{1}-2}=c 2^{p_{1}-1} \frac{z_{1}(a)}{z_{2}(b)^{p_{1}-1}}
$$

где $z_{1}(a)=\int_{0}^{1}\left(a^{2}+4 b^{2}\right)^{\frac{p_{1}-2}{4}} d b$ и $z_{2}(b)=\int_{0}^{1}\left(a^{2}+4 b^{2}\right)^{\frac{p_{2}-2}{4}} d a ;$ противоречие.

\section{3. Локальные свойства максимальной пары слоений}

ОПРЕДЕЛЕНИЕ 6. Пусть $\mathscr{F}_{1}, \mathscr{F}_{2}-k$-мерные слоения на открытых подмножествах $M$. Будем говорить, что $\mathscr{F}_{2}$ минорирует $\mathscr{F}_{1}$, если для любого $L_{1} \in \mathscr{F}_{1}$ существует $L_{2} \in \mathscr{F}_{2}$ такое, что $L_{2} \subset L_{1}$.

Ниже приводятся свойства модуля, которые являются сформулированными в терминах слоений аналогами свойствам из теоремы 1 в [11]. Часть из них отражает тот факт, что модуль является внешней мерой. 
Теорема 7. Пусть $\mathscr{F}_{1}, \mathscr{F}_{2}-k$-мерные слоения на открытых подмножествах $M$.

Пусть $p_{1}>0$.

1. Если $\mathscr{F}_{1} \subset \mathscr{F}_{2}$, то

$$
\bmod _{p_{1}}\left(\mathscr{F}_{1}\right) \leq \bmod _{p_{1}}\left(\mathscr{F}_{2}\right) .
$$

2. Если $\mathscr{F}_{1}$ минорирует $\mathscr{F}_{2}$, то

$$
\bmod _{p_{1}}\left(\mathscr{F}_{1}\right) \geq \bmod _{p_{1}}\left(\mathscr{F}_{2}\right) .
$$

Пусть $\mathscr{F}-$ слоение на $M$. Обозначим через $U_{i}, i=1, \ldots, \infty$, семейство взаимно не пересекающихся открытых подмножеств $M$ и $\mathscr{F}_{i}=\left.\mathscr{F}\right|_{U_{i}}$.

3. Если $\mathscr{F}_{\text {п. в. }}^{=} \bigcup_{i=1}^{\infty} \mathscr{F}_{i}$, то

$$
\bmod _{p_{1}}^{p_{1}}(\mathscr{F})=\sum_{i=1}^{\infty} \bmod _{p_{1}}^{p_{1}}\left(\mathscr{F}_{i}\right)
$$

Пусть $p_{1}>1$.

4. Если $\mathscr{F}$ минорируется всеми $\mathscr{F}_{i}$, то

$$
\bmod _{p_{1}}^{-p_{2}}(\mathscr{F}) \geq \sum_{i=1}^{\infty} \bmod _{p_{1}}^{-p_{2}}\left(\mathscr{F}_{i}\right)
$$

где $p_{1}, p_{2}$ сопряженные.

Пусть $\mathscr{F}$ и $\mathscr{G}$ - два ортогональных слоения комплементарных размерностей на $M$. Обозначим через $U_{j}^{i}, i \in 1, \ldots, m_{1}, j \in 1, \ldots, m_{2}\left(m_{1}, m_{2} \in \mathbb{N}\right)$, взаимно не пересекающиеся открытые подмножества $M$ такие, что $M \subset \bigcup_{i, j} \bar{U}_{j}^{i}$. Определим

$$
\begin{gathered}
\mathscr{F}_{j}^{i}=\left.\mathscr{F}\right|_{U_{j}^{i}}, \quad U^{i}=\operatorname{int}\left(\bigcup_{j} \bar{U}_{j}^{i}\right), \quad \mathscr{F}^{i}=\left.\mathscr{F}\right|_{U^{i}}, \quad \mathscr{G}^{i}=\left.\mathscr{G}\right|_{U^{i}}, \\
U_{j}=\operatorname{int}\left(\bigcup_{i} \bar{U}_{j}^{i}\right), \quad \mathscr{F}_{j}=\left.\mathscr{F}\right|_{U_{j}}, \quad \mathscr{G}_{j}=\left.\mathscr{G}\right|_{U_{j}} .
\end{gathered}
$$

Следующее утверждение существенно усиливает следствие 3.4 из [14].

Лемма 4. Предположим, что

(1) для любого $i$ слоение $\mathscr{F}^{i}$ минорируется $\mathscr{F}_{1}^{i}, \ldots, \mathscr{F}_{m_{2}}^{i}$ и $\mathscr{G}^{i} \underset{\text { п. в. }}{=} \bigcup_{j} \mathscr{G}_{j}^{i}$;

(2) для любого $j$ слоение $\mathscr{G}_{j}$ минорируется $\mathscr{G}_{j}^{1}, \ldots, \mathscr{G}_{j}^{m_{1}}$ и $\mathscr{F}_{j}=\bigcup_{\text {п. в. }} \mathscr{F}_{j}^{i}$.

Пусть пара сопряженных чисел $p_{1}, p_{2}$ и постоянные $a_{i, j}, i=1, \ldots, m_{1}$, $j=1, \ldots, m_{2}$, таковы, что

$$
\bmod _{p_{1}}\left(\mathscr{F}_{j}^{i}\right) \cdot \bmod _{p_{2}}\left(\mathscr{G}_{j}^{i}\right) \leq a_{i, j} .
$$

Тогда

$$
\bmod _{p_{1}}(\mathscr{F}) \cdot \bmod _{p_{2}}(\mathscr{G}) \leq \max _{i, j} a_{i, j}
$$

ДокАЗАТЕЛЬСТво. Согласно предположению (1) и свойствам 3, 4 из теоремы 7 имеем

$$
\frac{1}{\bmod _{p_{1}}^{p_{2}}\left(\mathscr{F}^{i}\right)} \geq \frac{1}{\bmod _{p_{1}}^{p_{2}}\left(\mathscr{F}_{1}^{i}\right)}+\cdots+\frac{1}{\bmod _{p_{1}}^{p_{2}}\left(\mathscr{F}_{m_{2}}^{i}\right)}
$$




$$
\bmod _{p_{2}}^{p_{2}}\left(\mathscr{G}^{i}\right)=\bmod _{p_{2}}^{p_{2}}\left(\mathscr{G}_{1}^{i}\right)+\cdots+\bmod _{p_{2}}^{p_{2}}\left(\mathscr{G}_{m_{2}}^{i}\right) .
$$

В силу вышеприведенных оценок получим следующую цепочку неравенств:

$$
\begin{aligned}
& \bmod _{p_{1}}^{p_{2}}\left(\mathscr{F}^{i}\right) \cdot \bmod _{p_{2}}^{p_{2}}\left(\mathscr{G}^{i}\right) \\
& \leq \frac{\bmod _{p_{1}}^{p_{2}}\left(\mathscr{F}_{1}^{i}\right) \cdots \cdot \bmod _{p_{1}}^{p_{2}}\left(\mathscr{F}_{m_{2}}^{i}\right)}{\bmod _{p_{1}}^{p_{2}}\left(\mathscr{F}_{2}^{i}\right) \cdots \cdot \ldots \cdot \bmod _{p_{1}}^{p_{2}}\left(\mathscr{F}_{m_{2}}^{i}\right)+\cdots+\bmod _{p_{1}}^{p_{2}}\left(\mathscr{F}_{1}^{i}\right) \ldots \cdot \bmod _{p_{1}}^{p_{2}}\left(\mathscr{F}_{m_{2}-1}^{i}\right)}\left(\bmod _{p_{2}}^{p_{2}}\left(\mathscr{G}_{1}^{i}\right)+\cdots+\bmod _{p_{2}}^{p_{2}}\left(\mathscr{G}_{m_{2}}^{i}\right)\right)
\end{aligned}
$$

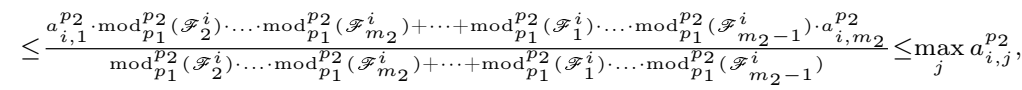

а значит,

$$
\bmod _{p_{1}}\left(\mathscr{F}^{i}\right) \cdot \bmod _{p_{2}}\left(\mathscr{G}^{i}\right) \leq a_{i},
$$

где $a_{i}=\max _{j} a_{i, j}$. Тогда в силу предположения (2) и свойств 3,4 теоремы 7 имеем

$$
\begin{aligned}
\frac{1}{\bmod _{p_{2}}^{p_{1}}(\mathscr{G})} & \geq \frac{1}{\bmod _{p_{2}}^{p_{1}\left(\mathscr{G}^{1}\right)}}+\cdots+\frac{1}{\bmod _{p_{2}}^{p_{1}}\left(\mathscr{G}^{m_{1}}\right)}, \\
\bmod _{p_{1}}^{p_{1}}(\mathscr{F}) & =\bmod _{p_{1}}^{p_{1}}\left(\mathscr{F}^{1}\right)+\cdots+\bmod _{p_{1}}^{p_{1}}\left(\mathscr{F}^{m_{1}}\right) .
\end{aligned}
$$

Те же шаги могут быть повторены далее для оценки $\bmod _{p_{1}}^{p_{1}}(\mathscr{F}) \cdot \bmod _{p_{2}}^{p_{1}}(\mathscr{G})$, что приведет к требуемому неравенству.

Следствие 3. В силу доказательства леммы 4 очевидно, что

$$
\bmod _{p_{1}}(\mathscr{F}) \cdot \bmod _{p_{2}}(\mathscr{G})<\max _{i, j} a_{i, j}
$$

если не все постоянные $a_{i, j}$ равны между собой.

Предыдущий результат порождает несколько следствий для максимальной пары слоений.

Следствие 4. Пусть $G: M \rightarrow R_{1} \times R_{2}$ - диффеоморфизм и $p_{1}, p_{2}-$ пара сопряженных чисел. Пусть слоения $\mathscr{F}_{1}, \mathscr{F}_{2}$, связанные диффеоморфизмом $\left(G ; R_{1}, R_{2}\right),\left(p_{1}, p_{2}\right)$-максимальны. Тогда для произвольных открытых множеств $R_{1}^{\prime} \subset R_{1}, R_{2}^{\prime} \subset R_{2}$ пара $\left.\mathscr{F}_{1}\right|_{G^{-1}\left(R_{1}^{\prime} \times R_{2}^{\prime}\right)},\left.\mathscr{F}_{2}\right|_{G^{-1}\left(R_{1}^{\prime} \times R_{2}^{\prime}\right)}$ также $\left(p_{1}, p_{2}\right)$ максимальна.

ДокАзАТЕЛЬСтво. Обозначим

$$
R_{1}^{\prime \prime}=\operatorname{int}\left(R_{1} \backslash R_{1}^{\prime}\right), \quad R_{2}^{\prime \prime}=\operatorname{int}\left(R_{2} \backslash R_{2}^{\prime}\right)
$$

и

$$
\begin{array}{ll}
U_{1}^{1}=G^{-1}\left(R_{1}^{\prime} \times R_{2}^{\prime}\right), & U_{2}^{1}=G^{-1}\left(R_{1}^{\prime} \times R_{2}^{\prime \prime}\right), \\
U_{1}^{2}=G^{-1}\left(R_{1}^{\prime \prime} \times R_{2}^{\prime}\right), & U_{2}^{2}=G^{-1}\left(R_{1}^{\prime \prime} \times R_{2}^{\prime \prime}\right) .
\end{array}
$$

Тогда $\mathscr{F}_{1}, \mathscr{F}_{2}$ и $\left.\mathscr{F}_{1}\right|_{U_{j}^{i}},\left.\mathscr{F}_{2}\right|_{U_{j}^{i}}, i, j=1,2$, удовлетворяют предположениям леммы 4. Значит, здесь может быть применено следствие 3: предположив, что утверждение не выполняется, получаем противоречие с максимальностью слоения.

Следующее утверждение можно считать эквивалентом свойства «локальной экстремальности» $p$-экстремальных функций для $p$-емкости четырехсторонника, которое обсуждалось в [17]. 
Теорема 8. Пусть $G: M \rightarrow R_{1} \times R_{2}$ - диффеоморфизм и $p_{1}, p_{2}$ - пара сопряженных чисел. Пусть слоения $\mathscr{F}_{1}, \mathscr{F}_{2}$, связанные диффеоморфизмом $\left(G ; R_{1}, R_{2}\right),\left(p_{1}, p_{2}\right)$-максимальны и $\int_{L_{1}} J G_{\mathscr{F}_{1}^{\perp}}^{\frac{1}{p_{1}-1}}<\infty$ для всех $L_{1} \in \mathscr{F}_{1}$. Тогда для любого открытого множества $R_{1}^{\prime} \subset R_{1}$ имеем

$$
f_{1}^{\prime}(x)=f_{1}(x)\left(\frac{\left\|f_{1}^{\prime}\right\|_{L^{p_{1}}\left(M^{\prime}\right)}}{\left\|f_{1}\right\|_{L^{p_{1}(M)}}}\right)^{p_{2}} \text { для } x \in M^{\prime}=G^{-1}\left(R_{1}^{\prime} \times R_{2}\right),
$$

где $f_{1}$ и $f_{1}^{\prime}-p_{1}$-экстремальные функции для $\mathscr{F}_{1}$ и $\left.\mathscr{F}_{1}\right|_{M^{\prime}}$ соответственно.

ДокАЗАтельство. Пусть $R_{1}^{\prime}$ - некоторое открытое подмножество $R_{1}$. По следствию 4 пара $\left.\mathscr{F}_{1}\right|_{M^{\prime}}$ и $\left.\mathscr{F}_{2}\right|_{M^{\prime}}$ также $\left(p_{1}, p_{2}\right)$-максимальна. Применяя теорему 4 к этим слоениям, а также к $\mathscr{F}_{1}, \mathscr{F}_{2}$, получаем существование соответствующих экстремальных функций $f_{1}^{\prime}, f_{2}^{\prime}, f_{1}, f_{2}$ наряду с соотношениями

$$
f_{2}^{\prime}(x)=\frac{f_{1}^{\prime}(x)^{p_{1}-1}}{\left\|f_{1}^{\prime}\right\|_{L^{p_{1}\left(M^{\prime}\right)}}^{p_{1}}}, \quad f_{2}(x)=\frac{f_{1}(x)^{p_{1}-1}}{\left\|f_{1}\right\|_{L^{p_{1}(M)}}^{p_{1}}} .
$$

Поскольку $\left.\mathscr{F}_{2}\right|_{M^{\prime}}$ состоит из целых листьев $\mathscr{F}_{2}$, в силу формулы $(4) f_{2}^{\prime}(x)=$ $f_{2}(x)$ для всех $x \in M^{\prime}$. Это равенство вместе с $(28)$ и $(7)$ приводит к требуемому утверждению.

\section{4. Заключительные замечания}

Обсуждение вопроса, является ли существование диффеоморфизма, удовлетворяющего набору условий, возникающих в следствии 2 , необходимым условием максимальности пары более чем одномерных слоений, как в случае семейства кривых в $\mathbb{R}^{2}$, определенных в четырехстороннике (см. [8, 17]), выходит за рамки данной статьи.

\section{ЛИТЕРАТУРА}

1. Ahlfors L. V. Lectures on quasiconformal mappings. Princeton, N.J.; Toronto; New York; London: Van Nostrand, 1966.

2. Gehring F. Quasiconformal mappings in space // Bull. Amer. Math. Soc. 1962. V. 69, N 2. P. $146-164$.

3. Heinonen J., Koskela P. Quasiconformal mappings in metric spaces with controlled geometry // Acta Math. 1998. V. 181. P. 1-61.

4. Väisälä J. Lectures on $n$-dimensional quasiconformal mappings. Berlin: Springer-Verl., 1971. (Lect. Notes Math.; V. 229).

5. HajŁasz P. Sobolev spaces on metric measure spaces // Contemp. Math. 2003. V. 338. P. 173-218.

6. Heinonen J. Nonsmooth calculus // Bull. Amer. Math. Soc., New Ser. 1979. V. 44, N 2. P. $163-232$.

7. Rodin B. The method of extremal length // Bull. Amer. Math. Soc. 1974. V. 80. P. 587-606.

8. Dymchenko $Y u$. V. Equality of the capacity and module of a condenser on a surface // J. Math. Sci. New York. 2003. V. 118, N 1. P. 4795-4807.

9. Hesse J. A $p$-extremal length and $p$-capacity equality // Ark. Mat. 1975. V. 13. P. 131-144.

10. Ziemer W. P. Extremal length and p-capacity // Michigan Math. J. 1969. V. 16. P. 43-51.

11. Fuglede B. Extremal length and functional completion // Acta Math. 1957. V. 98. P. 171-219.

12. Suominen K. Quasiconformal maps in manifolds // Ann. Acad. Sci. Fenn., Ser. AI. 1966. V. 393. P. 1-39.

13. Ciska M. Variation of the modulus of the foliation // J. Math. Anal. Appl. 2013. V. 401. P. 38-46.

14. Pierzchalski A. On the modulus of level sets of conjugate submersions // Differ. Geom. Appl. 2014. V. 36. P. 90-97. 
15. Kaźmierczak A., Pierzchalski A. On some criterion of conformality // Balk. J. Geom. Appl. 2016. V. 21, N 1. P. 51-57.

16. Pierzchalski A. The $k$-module of level sets of differential mappings // Math. Inst. Polish Acad. Sci. 1979. P. 180-185.

17. Романов А. С. Емкостные соотношения в четырехстороннике // Сиб. мат. журн. 2008. T. 49 , № 4. C. $886-897$.

Поступила в редакиию 12 марта 2019 г.

После доработки 12 марта 2019 г.

Принята к публикации 24 июля 2019 г.

Казмирчак Анна (Anna Kaźmierczak)

University of Lodz, Faculty of Mathematics and Computer Science,

Banacha 22, 90-238 Lodz, Poland

anna.kazmierczak@wmii.uni.lodz.pl 